\title{
NIR-responsive MXene nanobelts for wound healing
}

Lin Jin (1) ${ }^{1,2,3}$, Xiaoqing Guo ${ }^{4}$, Di Gao ${ }^{4}$, Cui Wu ${ }^{5}$, Bin Hu${ }^{3}$, Guangxuan $\operatorname{Tan}^{3}$, Nannan Du${ }^{3}$, Xiaolu Cai ${ }^{1,6}$, Zhe Yang $\mathbb{D}^{4,7}$ and Xingcai Zhang (5,8 $^{5,8}$

\begin{abstract}
Effectively achieving wound healing is a great challenge. Herein, we facilely prepared temperature-responsive MXene nanobelt fibers (T-RMFs) carrying vitamin E with a controllable release ability for wound healing. These T-RMFs were composed of MXene nanosheets spread along polyacrylonitrile and polyvinylpyrrolidone composite nanobelts together with a thermosensitive PAAV- coating layer. The high mass loading and high surface area of the MXene nanosheets endow the T-RMFs with excellent photothermal properties. The temperature could be easily controlled by near-infrared (NIR) irradiation exposure, and then the thermoresponsive polymeric coating layer relaxed the interface to dissolve vitamin $\mathrm{E}$ and promote vitamin $\mathrm{E}$ release. The T-RMFs demonstrated excellent biocompatibility and woundhealing functions in cellular and animal tests. The facile method, high mass loading, high surface area, excellent wound-healing functions, interesting nanosheet/nanobelt structure, mass production potential, and NIR responsive properties of these T-RMFs indicate the great potential of our nanobelts for wound healing, tissue engineering, and much broader application areas. This facile nanosheet/nanobelt preparation strategy paves a new way for nanomaterial fabrication and applications.
\end{abstract}

\section{Introduction}

As a typical clinical disease, wounds can occur from traumatic injury, diabetes, or burns, greatly affecting people's health and creating great issues in the medical and healthcare fields ${ }^{1-6}$. Although some treatments for wound healing, such as drug therapy ${ }^{7}$, skin grafting ${ }^{4,6,8}$, and cellular treatment ${ }^{9}$ have been used in clinical practice, there are still some limitations that reduce their therapeutic effects. Emerging nanofibrous scaffolds that can better mimic the natural extracellular matrix are ideal wound repair materials because they have superior biocompatibility and drug loading and effective interactions with the interface of the wound ${ }^{10-12}$. Various strategies have been used to develop functional scaffold-loaded

Correspondence: Lin Jin (jinlin_1982@126.com) or

Zhe Yang (yangzhe@xjtu.edu.cn) or Xingcai Zhang (xingcai@mit.edu)

${ }^{1}$ Henan Provincial People's Hospital, People's Hospital of Zhengzhou University,

Number 7 Weiwu road, Zhengzhou, Henan 450003, China

${ }^{2}$ The Fifth Affiliated Hospital of Guangzhou Medical University, Guangzhou, Guangdong 510700, China

Full list of author information is available at the end of the article drugs for rapid wound healing. These functional bioscaffolds are biocompatible, flexible, and capable of controllable release, demonstrating positive effects on wound healing. Although these scaffolds promote therapeutic effects, they only provide the unordered release of active factors and cannot achieve long-term and stable control release for wound areas, thus restricting their practical performance in biomedical areas.

MXenes, as a new class of two-dimensional (2D) transition metal materials, have attracted wide interest in the biomedical field owing to their unique properties ${ }^{13-16}$, such as their large specific surface area ${ }^{17}$, good electrical performance $^{18}$, and high photothermal conversion efficiency. ${ }^{19,20}$ Notably, MXene provides antibacterial properties, photothermal therapy, and fluorescent imaging properties for versatile biomedical applications ${ }^{20,21}$. To unleash their biomedical applications, MXenes need to be prepared with various architectures without changing their intrinsic attributes ${ }^{22-24}$. More importantly, MXene nanosheets can be degraded by the interaction between

\section{(c) The Author(s) 2021}

(c) (i) Open Access This article is licensed under a Creative Commons Attribution 4.0 International License, which permits use, sharing, adaptation, distribution and reproduction cc) in any medium or format, as long as you give appropriate credit to the original author(s) and the source, provide a link to the Creative Commons license, and indicate if changes were made. The images or other third party material in this article are included in the article's Creative Commons license, unless indicated otherwise in a credit line to the material. If material is not included in the article's Creative Commons license and your intended use is not permitted by statutory regulation or exceeds the permitted use, you will need to obtain permission directly from the copyright holder. To view a copy of this license, visit http://creativecommons.org/licenses/by/4.0/. 
water and oxygen. Therefore, it is feasible to employ these unique photothermal properties and biodegradable performance for wound healing. However, it is a considerable challenge to develop MXene nanosheet-based nanofibrous scaffolds because of the irregular shape and size of the exfoliated MXene nanosheets.

$\mathrm{P}(\mathrm{AAm}-\mathrm{co}-\mathrm{AN}-\mathrm{CO}-\mathrm{VIm})$ copolymer (PAAV) is a copolymer that is synthesized by radical polymerization and displays unique temperature sensitivity ${ }^{25}$. Using PAAV as the surface carrier of the scaffold can effectively control the positive factor release rate by temperature regulation, which has the potential for long-term stable drug release.

In this study, we developed MXene fibrous nanosheets with a unique structure and temperature sensitivity for wound healing. The obtained RMFs have a large width to thickness ratio. The MXene nanosheets showed suitable wetting and spreading effects, allowing easy contact with the surface of the skin to exert its photothermal properties. Furthermore, the temperature-responsive MXene nanobelt fibers (T-RMFs) demonstrated good biocompatibility and could provide an excellent microenvironment for cell survival. More importantly, the capacity of the T-RMFs to deliver drugs controllably with responsive performance provides a favorable strategy for wound healing. Considering the highly enriched integration of these unique structural and photothermal properties, we hope that the prepared T-RMFs could advance wound healing for damaged skin.

\section{Experimental section}

Materials: polyvinylpyrrolidone (PVP) $(\mathrm{MW}=30 \mathrm{kD})$ and polyacrylonitrile $(\mathrm{PAN})(\mathrm{MW}=10 \mathrm{kD})$ were purchased from Daigang Polymer (Jinan, China). All other chemicals were purchased from Guangzhou Chemical Co. (Guangzhou, China) and used without further purification.

\section{Fabrication of the T-RMFs}

First, we prepared modified MXene nanosheets ${ }^{26}$. The obtained MXene nanosheets were dispersed in $3 \mathrm{~mL}$ of DMF solution at a concentration of $120 \mathrm{mg} / \mathrm{mL}$, and then $240 \mathrm{mg}$ of PAN and $240 \mathrm{mg}$ of PVP were added into $3 \mathrm{~mL}$ of the MXene nanosheets and DMF mixed solution followed by stirring for $24 \mathrm{~h}$. Finally, the mixed solution was transferred into a syringe with a needle, and we prepared the RMFs by our electrospinning technique ${ }^{27,28}$. A high voltage of $15 \mathrm{kV}$ was used for the electrospinning process, with a $15 \mathrm{~cm}$ collector-to-needle distance.

The T-RMFs were prepared by coating the PAAV layer through the RMFs soaked in PAAV aqueous solution at $80^{\circ} \mathrm{C}$ for $1 \mathrm{~h}$ and then dried in a drier at $60^{\circ} \mathrm{C}$.

The temperature sensitivity of the shell could result in the open or close of the positive factor in the carrier, which is very important for maintaining long-term and stable drug release. PAAV is a copolymer that is synthesized by radical polymerization and displays unique temperature sensitivity ${ }^{25}$. Therefore, using PAAV as the carrier of the scaffold can effectively control the rate of release by temperature regulation.

As reported in the previously synthesized method ${ }^{25}$, the monomers (1-vinyl-imidazole, acrylonitrile, acrylamide), initiator (AIBN), and chain transfer agent (thioglycolic acid) were dissolved in dimethylformamide and moved to a Schlenk flask. For this reaction, the temperature remained at $65^{\circ} \mathrm{C}$, and the reaction time was $24 \mathrm{~h}$. The PAAV copolymer was obtained by precipitation and purification.

\section{Morphology and chemical characterization of the RMFs}

The morphology of the rod-like MXene fibers in the T-RMFs and RMFs was studied by transmission electron microscopy (TEM) and scanning electron microscopy (SEM).

\section{Photothermal conversion of the RMFs}

The photothermal performance of T-RMFs and RMFs exposed to the near-infrared (NIR) laser is shown by the temperature evolution recorded using an infrared thermal imager (Fluke TIS 20+). In brief, T-RMFs and RMFs in $0.5 \mathrm{~mL}$ of ultrapure water were added to 24-well plates followed by exposure to an $808 \mathrm{~nm}$ NIR laser at $0.33,0.5$, and $1 \mathrm{~W} / \mathrm{cm}^{2}$. To measure the photothermal stabilities of the RMFs, each sample was irradiated with an $808 \mathrm{~nm}$ laser five times, and the temperature changes during this process were recorded.

\section{Cell culture and evaluation}

BMSCs were used to test the cellular response of the cells and fibers of T-RMFs and RMFs. PAN-PVP nanofibers were prepared and treated using the abovementioned method as a control group ${ }^{27}$. The cellular viability and morphology between the BMSCs and fibrous fiber surface of the T-RMFs and RMFs were tested using a previous method ${ }^{28}$.

\section{In vivo wound-healing studies}

We carried out in vivo wound-healing studies using a full-thickness skin defect model (male Kunming mice (20-30 g, 5-6 weeks of age)). First, all mice were randomly divided into five groups. These five groups contained six mice each and were the control (Tegaderm film), RMF, RMF with laser, T-RMF, and T-RMF with laser groups. All surgical procedures were performed under aseptic conditions. Next, the mice were anesthetized using an intraperitoneal injection of 5\% chloral hydrate solution $(10 \mathrm{~mL} / \mathrm{kg}$ body weight) and shaved in the dorsal region between the tail and back. The fullthickness wounds were formed by creating a punch with a 
diameter of $\sim 8 \mathrm{~mm}$. Then, the wounds in the control group had only the Transparent Film Dressing Frame Style added. For the sample groups, RMFs or T-RMFs $(1 \mathrm{~cm}$ in diameter) were applied to the wounds. On the 1 st, 3rd, 7th, 10th, 14th, and 17th days, all wound areas were measured and analyzed.

For the immunohistochemical study, on the 17th day, the skin containing the wounds was collected (diameter $=$ $1 \mathrm{~cm}$ ) and fixed in tissue fixative. The CD31, VEGF, and PCNA levels were evaluated by immunofluorescent staining. All operations followed the manufacturer's instructions.

\section{Results and discussion}

Production and chemical and morphological studies of the T-RMFs

The fabrication scheme of the T-RMFs is shown in Fig. 1. MXene nanosheets, as an important part of the TRMFs, were fabricated first ${ }^{25,26}$. AFM and TEM images are shown in Fig. S1A and Fig. S1B and indicated that the obtained MXene nanosheets had a good size distribution $(0.5-3 \mu \mathrm{m})$ and thickness $(1.2 \mathrm{~nm})$. In addition, good monolayer dispersion was clearly observed (Fig. S1C).

Next, we used an electrospinning process to fabricate the RMFs. SEM images of the raw prepared PAN-PVP fibers are shown in Fig. S2, which indicated that the morphology of the PAN-PVP fibers were not obviously different than the other previously fabricated fibers; only the diameter was larger. However, after adding a high concentration $(80 \mathrm{mg} / \mathrm{mL})$ of the MXene nanosheets, the morphology of the RMFs showed great changes compared with traditional fibers (Fig. S3). The RMFs displayed obvious rod-like structures (Fig. 2A, B) with a size of $2.5 \pm$ $0.5 \mu \mathrm{m}$, thickness of $50 \pm 20 \mathrm{~nm}$, and a width to thickness

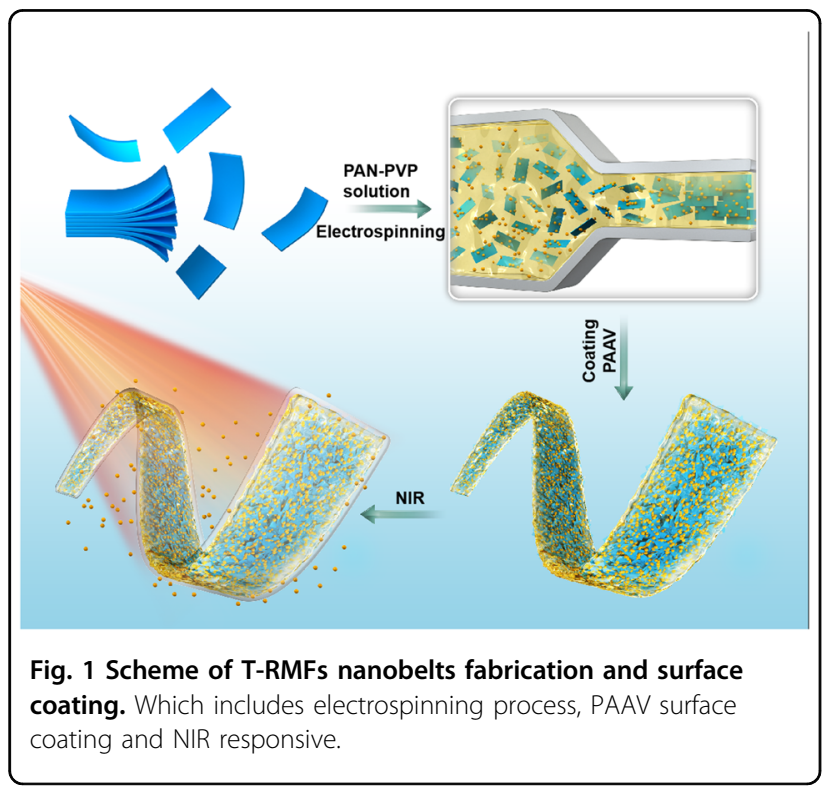

ratio of up to 50 . This special structure provides a large contact area, which has an important role in drug loading and release. The TEM image of a single RMF belt (Fig. S4) clearly showed the MXene nanosheets in the fibrous band. After coating with the PAAV layer, the surface of the fibers in the RMFs had some changes. Although the morphology remained good, the RMFs displayed obvious winkles (Fig. 2D). In addition, the PAAV surface layer was clearly observed (Fig. 2 and Fig. S5).

For chemical characterization of the RMFs, the mapping pattern (Fig. 2C) showed related elements, such as Ti, N, and $\mathrm{C}^{29}$ and the energy-dispersive $\mathrm{X}$-ray spectroscopy pattern (Fig. S6) displayed MXene nanosheets in the fibrous band ${ }^{30}$. In addition, the XRD pattern showed characteristic MXene peaks (Fig. 2F) ${ }^{31}$. These results indicated that the MXene nanosheets were successfully embedded into the fibrous band.

\section{T-RMFs photothermal conversion}

Under NIR exposure, the prepared MXene-doped fiber belts can generate heat. The test results indicated that when the NIR light power was $1.0 \mathrm{~W}$, the temperature rapidly reached $60^{\circ} \mathrm{C}$ in $2 \mathrm{~min}$, whereas the highest temperature that the T-RMFs can be is $65^{\circ} \mathrm{C}$ (Fig. 3A). As the NIR power was reduced to $0.5 \mathrm{~W}$, the temperature also increased to $42{ }^{\circ} \mathrm{C}$ within $3 \mathrm{~min}$. The peak thermal images at different powers are shown in Fig. 3B. As the NIR power was reduced to $0.33 \mathrm{~W}$ (a level safe for applications), the temperature could reach $41{ }^{\circ} \mathrm{C}$ in $3 \mathrm{~min}$. Considering that high temperature might be harmful to the nearby skin, $0.33 \mathrm{~W}$ was selected for practical application. The RMFs and T-RMFs were tested with five consecutive NIR on/off cycles. For every cycle, the obtained RMFs could be heated to $41^{\circ} \mathrm{C}$ (Fig. 3B), and when the NIR irradiation was switched off, the temperature immediately decreased to the initial value. Importantly, after coating with PAAV, the RMFs remained photothermally similar, and the T-RMFs quickly heated up to $39^{\circ} \mathrm{C}$ (Fig. 3C). This result showed that the T-RMFs could maintain unique photothermal conversion. In summary, our study indicated that the T-RMFs could be repetitively controlled by NIR exposure. Moreover, the RMFs and T-RMFs displayed excellent photothermal effects on mouse dorsal skin. The temperatures of the RMF and T-RMF model areas increased from $\sim 23^{\circ} \mathrm{C}$ (Fig. 3D) to $\sim 41^{\circ} \mathrm{C}$ (Fig. 3E) and $39^{\circ} \mathrm{C}$ (Fig. 3F) in $3 \mathrm{~min}$, respectively, and the results indicated that the RMFs and T-RMFs had desirable heating performance in vivo.

\section{Biocompatibility of the T-RMFs}

To evaluate the biocompatibility of the obtained T-RMFs with their spatial structure, a Cell Counting Kit-8 assay was used to evaluate cellular cytotoxicity. RMFs and PAN-PVP nanofibers were used as control 


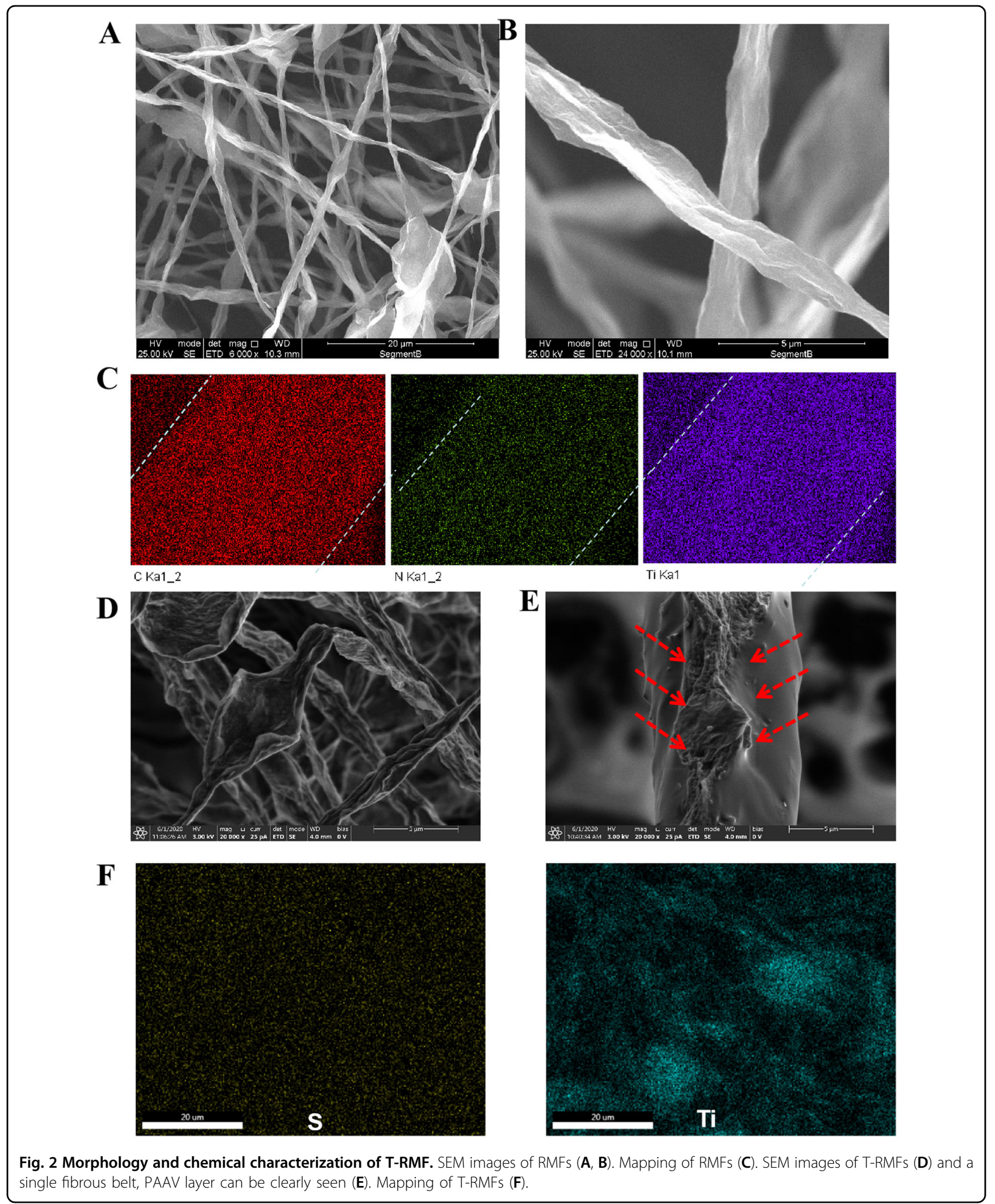

groups. BMSCs were cultured and incubated for evaluation. The cell evaluation results noted that the cells on the T-RMFs had much better cell attachment and proliferation than those on the control fibers during the culture period (Fig. 4C). These results indicated that the high-density MXene and PAAV coating layer of 

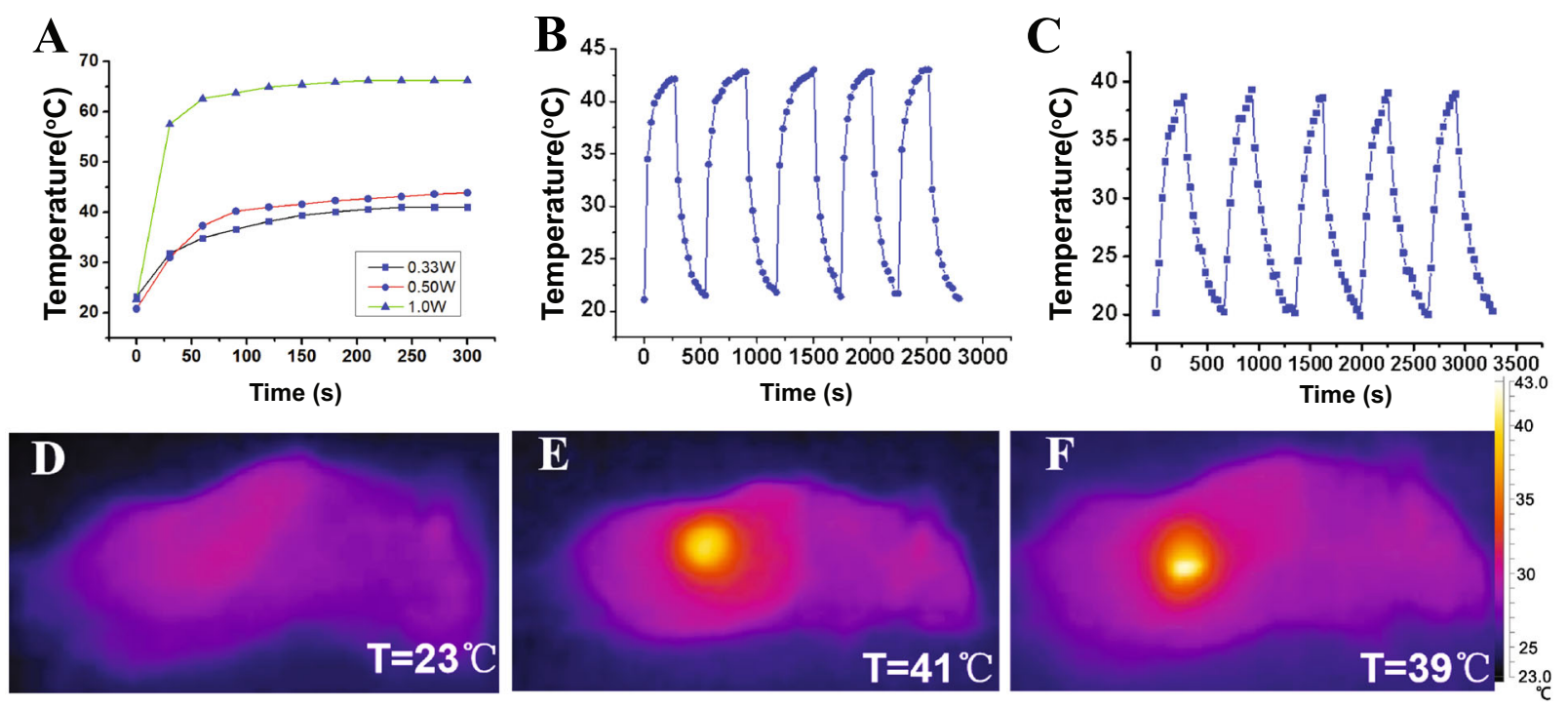

Fig. 3 Photothermal conversion of the RMFs and T-RMFs. Temperature increase profiles of the RMFs under $0.33 \mathrm{~W}, 0.50 \mathrm{~W}$, and 1.0 W of NIR light (A). Temperature changes of the RMFs (B) and T-RMFs (C) during five on/off cycles. Thermal images of the control group (D), RMFs (E) and T-RMFs (F) under 0.33 W of NIR light.

the prepared T-RMFs have excellent biocompatible properties.

Fluorescent images (Fig. 4A) of the cells cultured on the T-RMFs, RMFs, and PAN-PVP indicated that BMSCs displayed excellent cellular activity and grew along the fibrous belts. In addition, the cellular morphology of the BMSCs on the T-RMFs demonstrated a widespread along the fibrous belts and formed integrated cell-fiber constructs (Fig. 4B) that were attributed to the amino, hydroxyl, and carboxyl groups on the surface of the TRMFs, which provided the desired microenvironment for cell growth. Moreover, we observed that the surface of the nanofibers on the RMFs was slightly changed owing to the MXene nanosheets absorbed by the BMSCs. In contrast, cells in the PAN-PVP nanofiber group demonstrated a significant contraction. These results indicated that T-RMFs could provide the required elements or functional groups for cellular growth and proliferation while also supplying an excellent spatial structure for the cells to achieve excellent cellular activity.

Based on the excellent photothermal conversion properties of the T-RMFs, the PAAV layer could be opened, and the PVP glass transition temperature was reached after NIR treatment. Then, vitamin E was released from fibrous fibers. Notably, as more vitamin E can be stably released over a longer period of time through NIR exposure, greater wound-healing capacity promotion will be achieved.

Considering feasibility, the effective intermittent NIR irradiation of the T-RMFs was selected to be $3 \mathrm{~min}$ to achieve adequate vitamin E supply. After NIR irradiation, some pores were observed in the SEM image (Fig. S7) owing to the dissolved PVP in PBS solution, which indicated that PVP displayed dissolvability at high temperature. To evaluate the applications of the T-RMFs, $1 \mathrm{~cm}$ round wound models were established using mice. The mice were divided into five groups, and one group did not receive therapy while the other four groups were treated with T-RMF (PAAV+MXene+NIR group), RMF (MXene+NIR group), or RMF (MXene) or was a blank group; the T-RMF (PAAV+MXene+NIR group) and RMF (MXene+NIR group) groups received MXene nanosheets, vitamin $\mathrm{E}$ application, and added regular NIR irradiation (MXene+vitamin E NIR). Thermal imaging of the mice treated with the RMFs and T-RMFs indicated that the temperature could increase quickly after NIR exposure (Fig. S8). The healing conditions of the wound areas were observed at predetermined times. The T-RMF $+\mathrm{NIR}, \mathrm{RMF}+\mathrm{NIR}$, and RMF groups displayed much better recovery than the control groups. In particular, the wounds on the mice in the T-RMF+NIR group were almost healed on day 17 (Fig. 5A). In addition, wound bed constriction and epithelization conditions were recorded and analyzed using hematoxylin-eosin (H\&E) staining. These results indicated that the wounds in the T-RMF +NIR group with vitamin E under NIR exposure contracted faster than the other groups and the new epithelial tissues in this treatment group were much thicker (Fig. 5B), indicating that the provided healing conditions allowed the long-term and stable release of vitamin $E$. The RMF group displayed worse recovery than the control group, which may be because the RMFs limited the flow 


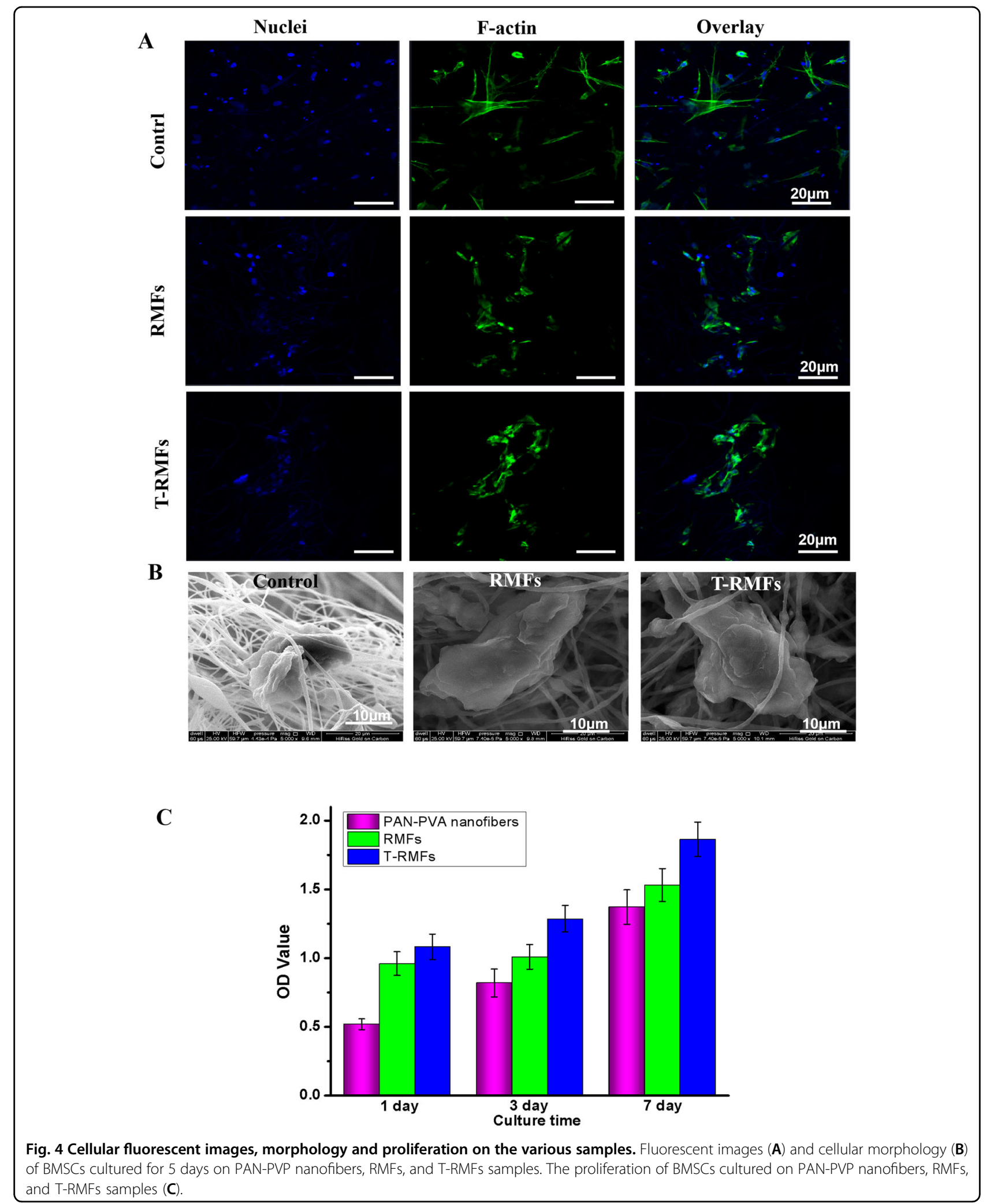

of oxygen. In contrast, the RMF group also treated with regular NIR irradiation showed an improved healing process, which may be attributed to the vitamin E release, swelling, and contraction of the polymer that promotes the flow of oxygen. More importantly, the T-RMF group with NIR exposure displayed the best therapeutic effects. 


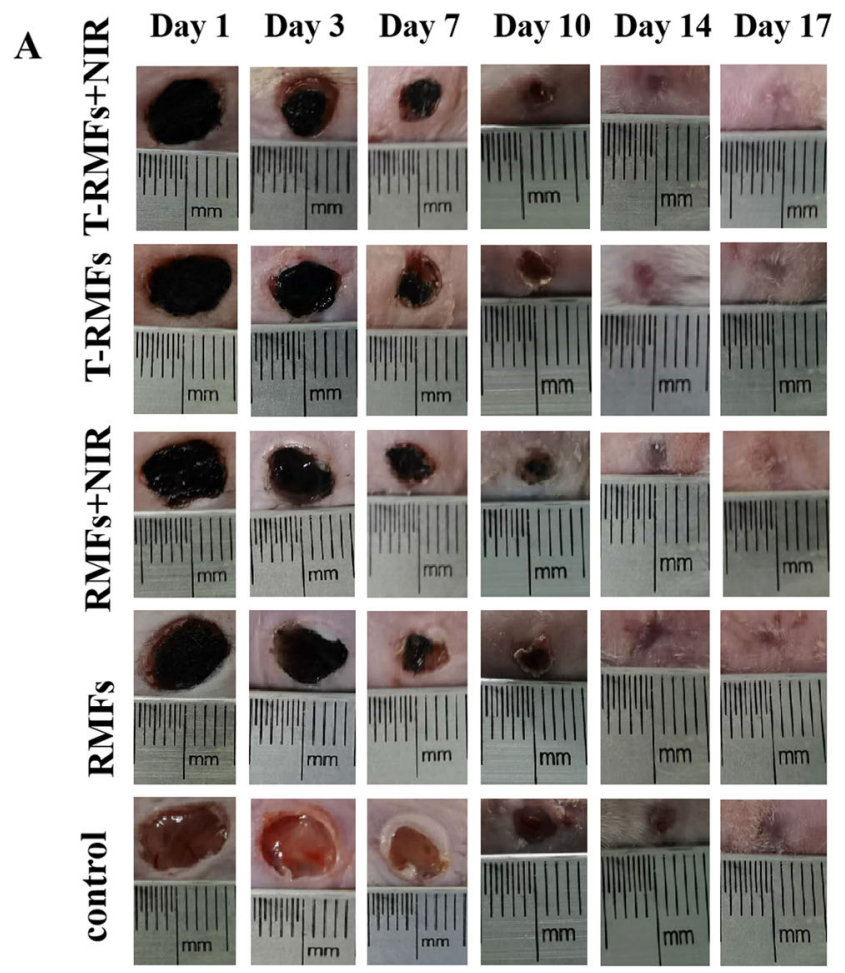

B
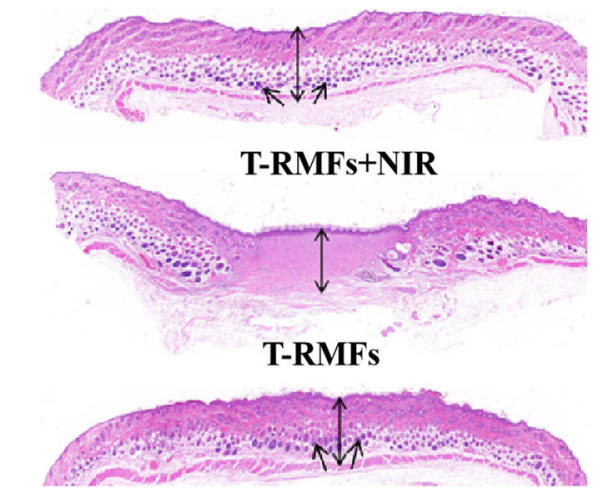

RMFs+NIR
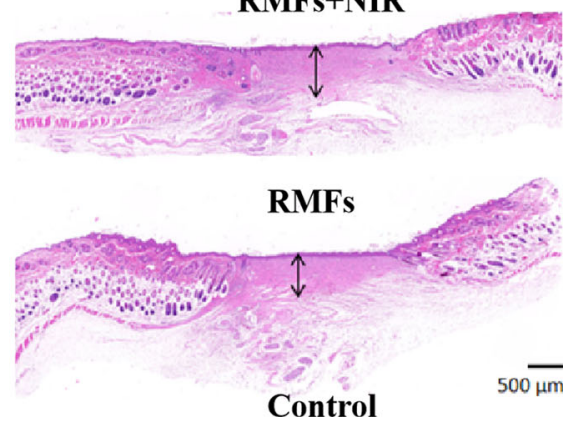

Fig. 5 Evaluation of the response of T-RMFs to wound healing in mice. Representative photographs of the skin wounds in various groups (control group, RMF group, RMF + NIR group, T-RMF group, and T-RMF + NIR group) on days 1, 3, 7, 10, 14, and 17. Scale bars are $1.0 \mathrm{~cm}$ (A). Corresponding H\&E staining images of the wound beds on day 17. Scale bars are $500 \mu \mathrm{m}$ (B).

For example, the wound area was the smallest, the new epithelial tissue was the thickest, the wound bed was the narrowest and the healing effect was the best compared with the RMF under NIR group (Fig. S9). This result showed that the PAAV layer could be loosened at high temperatures and closed when the temperature was low, providing effective control of vitamin E release for highly efficient application. These results indicated that NIR exposure, an effective surface layer temperature response, provided stable and long-term vitamin $\mathrm{E}$ release to achieve good wound healing.

Immunohistochemical staining of CD31 and $\alpha$-smooth muscle actin ( $\alpha$-SMA) was used to evaluate the woundhealing mechanism. The results indicated that the T-RMF + NIR group displayed increased CD31 expression (Fig. $6 \mathrm{~A}, \mathrm{D})$ and substantial angiogenesis. Furthermore, the RMF group with NIR exposure showed higher CD31 expression and angiogenesis than the RMF group without NIR exposure, indicating that adequate nutrient and oxygen supply benefits cytokine and cell transportation. The activation of angiogenesis is necessary for producing new tissues. As shown in the immunofluorescent staining in Fig. 6B, E, in the vitamin E+NIR treatment groups, the blood vessel density in the wound was greatly increased. Compared to the group without NIR exposure, the T-RMF+NIR and RMF+NIR groups also displayed better therapeutic effects (faster wound closure, angiogenesis, and tissue regeneration). The effects of wound healing can also be reflected by cell proliferation, which was detected by the immunohistochemical staining of PCNA, a substance that induces and participates in DNA synthesis. Figure $6 \mathrm{C}, \mathrm{F}$ demonstrates that the dual functions of photothermal conversion and vitamin $\mathrm{E}$ treatment in the T-RMFs significantly promoted cell multiplication, which was undoubtedly the best among the three groups. All of the results demonstrated that the T-RMFs had a desirable responsive capacity for wound healing. In addition, safety to the heart, spleen, liver, lung, and kidney was evaluated by $H \& E$ staining, and the results indicated that these organs remained normal, similar to no treatment (Fig. S10).

\section{Conclusions}

In summary, we developed a class of NIR- and temperature-responsive T-RMFs that had controllable drug delivery and repair capacity for wound healing. The excellent photothermal effects of the T-RMFs could better meet the complicated needs for healing wounds in clinical practice applications. The MXene nanosheets in these fibrous mats not only endowed the T-RMFs with excellent 


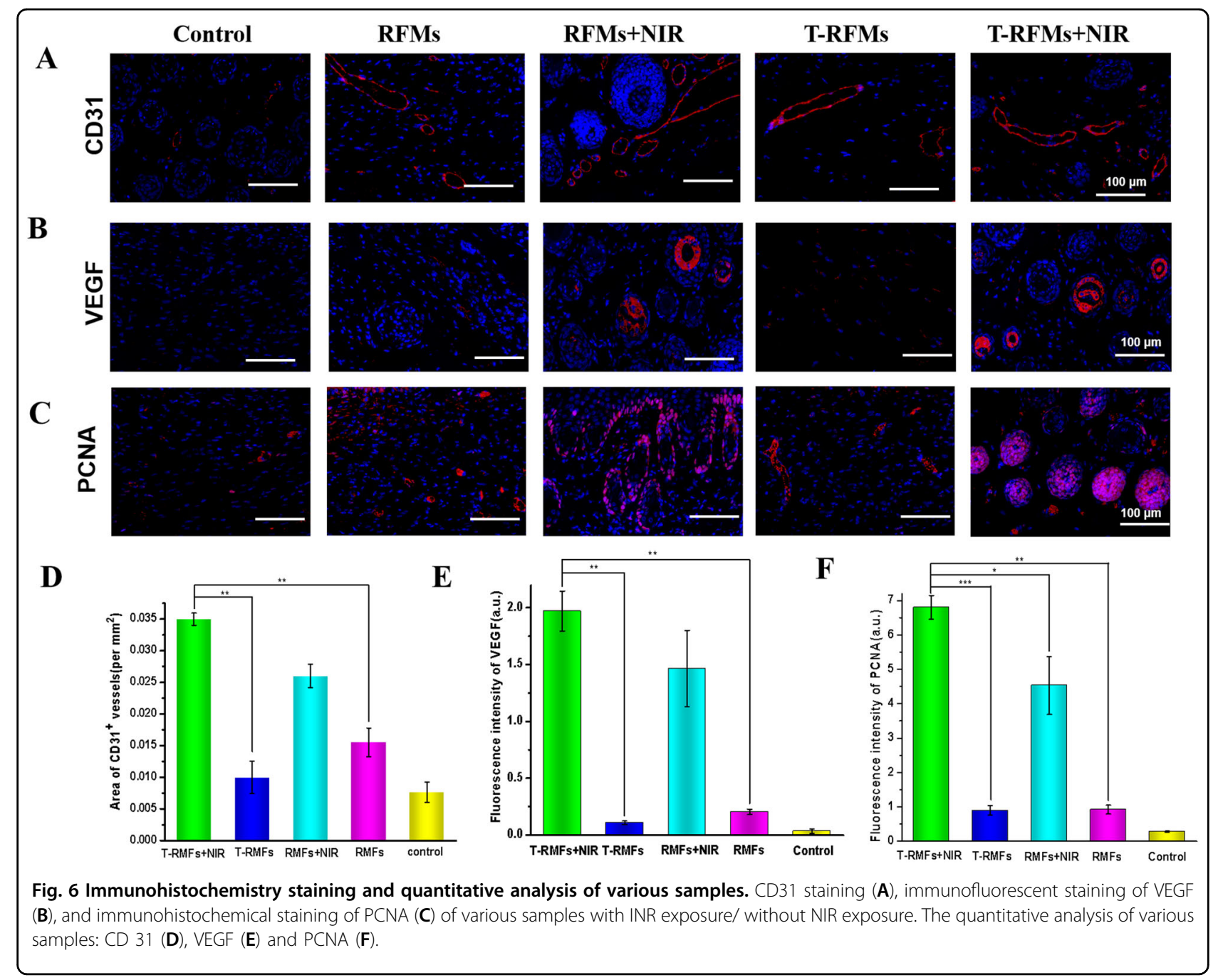

photothermal effects but also provided functional groups for cell growth. Furthermore, the PAAV layer, as a temperature-responsive factor, displayed an excellent controllable drug release ability and greatly facilitated long-term stable wound healing. The facile method, high mass loading, high surface area, excellent wound-healing functions, interesting nanosheet/nanobelt structure, mass production potential, and NIR responsive properties of the T-RMFs indicate the great potential of our nanobelts for wound healing, drug release, tissue engineering, biosensors, and much broader application areas ${ }^{32-39}$. The facile nanosheet/nanobelt preparation strategy paves a new way for nanomaterial fabrication and applications ${ }^{40}$.

\section{Acknowledgements}

This research was supported by the National Natural Science Foundation of China (U1904176, 51703178, 51903203, 51903258), Zhongyuan Thousand Talents Plan Project, Biomedicine Joint Fund of Zhe Jiang Province (LWY20H180002), Guangdong Natural Science Foundation Project (2019A1515010222), Guangzhou Municipal Science and Technology Project (201904010437), Henan Province Medical Science and Technology Research Project jointly built by the Ministry and Province (no. SB201901073), and Basic
Research Project of Key Scientific Research Projects in Henan Province (no. 20zx011).

\section{Author details}

${ }^{1}$ Henan Provincial People's Hospital, People's Hospital of Zhengzhou University, Number 7 Weiwu road, Zhengzhou, Henan 450003, China. ${ }^{2}$ The Fifth Affiliated Hospital of Guangzhou Medical University, Guangzhou, Guangdong 510700, China. ${ }^{3}$ International Joint Research Laboratory for Biomedical Nanomaterials of Henan, Zhoukou Normal University, Zhoukou, Henan 466001, China. ${ }^{4}$ The Key Laboratory of Biomedical Information Engineering of Ministry of Education, School of Life Science and Technology, Xi'an Jiaotong University, Xi'an, Shaanxi 710049, China. ${ }^{5}$ School of Engineering and Applied Sciences, Harvard University, Cambridge, MA 02138, USA. ${ }^{6}$ College of Chemistry, Zhengzhou University, Zhengzhou, Henan 450001, China. ${ }^{7}$ Research Institute of Xi'an Jiaotong University, Hangzhou, Zhejiang 311200, China. ${ }^{8}$ School of Engineering, Massachusetts Institute of Technology, Cambridge, MA 02139, USA

\section{Conflict of interest}

The authors declare no competing interests.

\section{Publisher's note}

Springer Nature remains neutral with regard to jurisdictional claims in published maps and institutional affiliations. 
Supplementary information The online version contains supplementary material available at https://doi.org/10.1038/s41427-021-00289-w.

Received: 24 October 2020 Revised: 28 December 2020 Accepted: 19 January 2021.

Published online: 8 March 2021

\section{References}

1. Mahmoudi, S. et al. Heterogeneity in old fibroblasts is linked to variability in reprogramming and wound healing. Nature 574, 553-5583 (2019).

2. Tetley, R. J. et al. Tissue fluidity promotes epithelial wound healing. Nature $\mathbf{1 5}$ 1195-1203 (2019)

3. Zhang, X. X. et al. Black phosphorus-loaded separable microneedles as responsive oxygen-delivery carriers for wound jealing. ACS Nano 14, 5901-5908 (2020)

4. Hou, R. et al. Surface-degradable drug-eluting stent with anticoagulation, antiproliferation, and endothelialization functions. Biomolecules 92, 69 (2019).

5. Hao, Y. L. et al. Effective wound healing enabled by discrete alternative electric fields from wearable nanogenerators. ACS Nano 12, 12533-12540 (2018).

6. Wang, X. et al. Differentiation of bMSCs on biocompatible, biodegradable, and biomimetic scaffolds for largely defected tissue repair. ACS Appl. Bio Mater. $\mathbf{3}$, 735-746 (2019).

7. Ouyang, J. et al. In situ sprayed NIR-responsive, analgesic black phosphorusbased gel for diabetic ulcer treatment. Proc. Natl Acad. Sci. USA 117 28667-28677 (2020)

8. Cui, T. T. et al. Large-scale fabrication of robust artificial skins from a biodegradable sealant-loaded nanofiber scaffold to skin tissue via microfluidic blowspinning. Adv. Mater. 32, 2000982 (2020).

9. Srifa, W. et al. Cas9-AAV6-engineered human mesenchymal stromal cells improved cutaneous wound healing in diabetic mice. Nat. Commun. 11, 2470 (2020).

10. Stapelfeldt, $\mathrm{K}$. et al. Controlling the multiscale structure of nanofibrous fibrinogen scaffolds for wound healing. Nano Lett. 19, 6554-6563 (2019).

11. Wang, S. Q. et al. Nanoenzyme-reinforced injectable hydrogel for healing diabetic wounds infected with multidrug resistant bacteria. Nano Lett. 20, 5149-5158 (2020)

12. Li, Z. et al. Biomimicry, biomineralization, and bioregeneration of bone using advanced three-dimensional fibrous hydroxyapatite scaffold. Mater. Today Adv. 3, 100014 (2019).

13. Kamaysbayew, V. et al. Covalent surface modifications and superconductivity of two-dimensional metal carbide MXenes. Science 369, 979-983 (2020).

14. Zhao, X. et al. Smart Ti3C2TX MXene fabric with fast humidity response and joule heating for healthcare and medical therapy applications. ACS Nano 14, 8793-8805 (2020).

15. Li, Z. K. et al. Antibiotics separation with MXene membranes Based on regularly stacked high-aspect-ratio nanosheets. Angew Chem. Int. Ed. 59, 9751-9756 (2020)

16. Xu, D. X., Li, Z. D., Li, L. S. \& Wang, J. Insights into the photothermal conversion of 2D MXene nanomaterials: synthesis, mechanism, and applications. Adv. Funct. Mater. 30, 2000712 (2020).

17. Wang, C. D., Chen, S. M. \& Song, L. Tuning 2D MXenes by surface controlling and interlayer engineering: methods, properties, and synchrotron radiation characterizations. Adv. Funct. Mater. 30, 2000869 (2020).
18. Shin, W. E. H. et al. Large-scale wet-spinning of highly electroconductive MXene fibers. Nat. Commun. 11, 2825 (2020).

19. Lao, J. C. et al. Electricity generation based on a photothermally driven Ti3C2TX MXene nanofluidic water pump. Nano Energy 70, 104481 (2020).

20. Gao, D. et al. Multifunctional phototheranostic nanomedicine for cancer imaging and treatment. Mater. Today Bio 5, 100035 (2020).

21. Pan, S. S. et al. 2D MXene-integrated 3D-printing scaffolds for augmented osteosarcoma phototherapy and accelerated tissue reconstruction. Adv. Sci. 7, 1901511 (2020).

22. Gao, Y. Y. et al. Microchannel-confined MXene based flexible piezoresistive multifunctional micro-force sensor. Adv. Funct. Mater. 30, 1909603 (2020).

23. Liao, H., Guo, X. L., Wan, P. B. \& Yu, G. H. Conductive MXene nanocomposite organohydrogel for flexible, healable, low-temperature tolerant strain sensors. Adv. Funct. Mater. 29, 19040507 (2019).

24. Xia, Y. et al. Thickness-independent capacitance of vertically aligned liquidcrystalline MXenes. Nature 557, 409-412 (2018).

25. Yang, Z. et al. Fighting immune cold and reprogramming immunosuppressive tumor microenvironment with red blood cell membrane-camouflaged nanobullets. ACS Nano 14, 17442-17457 (2020).

26. Huang, R. et al. MXene composite nanofibers for cell culture and tissue engineering. ACS Appl. Bio Mater. 3, 2125-2131 (2020).

27. Jin, L. et al. Synergistic effects of electrical stimulation and aligned nanofibrous microenvironment on growth behaviour of mesenchymal stem cells. ACS Appl. Mater. Interfaces 10, 18543-18550 (2018).

28. Jin, L. et al. Fabrication and characterization of three-dimensional (3D) coreshell structure nanofibers designed for 3D dynamic cell culture. ACS Appl. Mater. Interfaces 9, 17718-17726 (2017).

29. Li, X. X. et al. Self-healing microsupercapacitors with size-dependent 2D MXene. Adv. Mater. 7, 821-829 (2020).

30. Shen, J. et al. $2 \mathrm{D}$ MXene nanofilms with tunable gas transport channels. Adv Funct. Mater. 28, 1801511 (2018).

31. Umrao, S. et al. MXene artificial muscles based on ionically cross-linked Ti3C2Tx electrode for kinetic soft robotics. Sci. Robot. 4, 7797 (2019).

32. Parekh, G., Shi, Y., Zheng, J., Zhang, X. \& Leporatti, S. Nano-carriers for targeted delivery and biomedical imaging enhancement. Ther. Deliv. $\mathbf{9}$ 451 (2018).

33. Gong, Z. et al. Composite nanomaterial thin film-based biosensors. SENSORS, 2010 IEEE, 29-32 (2010)

34. Han, F. et al. Triple-synergistic $2 \mathrm{D}$ material-based dual-delivery antibiotic platform. NPG Asia Mater. 12, 15 (2020).

35. Yang, J. et al. Biologically modified nanoparticles as theranostic bionanomaterials. Prog. Mater. Sci. 100768 (2020)

36. Li, J., Li, Z., Chu, D., Jin, L. \& Zhang, X. Fabrication and biocompatibility of core-shell structured magnetic fibrous scaffold. J. Biomed. Nanotechnol. 15, 500-506 (2019).

37. Tang, Z. et al. A materials-science perspective on tackling COVID-19. Nat. Rev. Mater. 26, 458 (2020).

38. Zhou, M. et al. pH-sensitive poly ( $\beta$-amino ester) s nanocarriers facilitate the inhibition of drug resistance in breast cancer cells. Nanomaterials $\mathbf{8}$ 952 (2018).

39. Tang, Z. et al. Insights from nanotechnology in COVID-19 treatment. Nano Today 36, 101019 (2020).

40. Mai, W. et al. A versatile bottom-up interface self-assembly strategy to hain nanoparticle-based 2D monolayered composite and functional nanosheets. Chem. Comm. 55, 10241 (2019). 